\title{
Multicomponent solution in modified theory of gravity in the early universe
}

\author{
H. Mohseni Sadjadi* \\ Department of Physics, University of Tehran , \\ P. O. B. 14395-547, Tehran 14399-55961, Iran
}

August 21, 2021

\begin{abstract}
We study the modified theory of gravity in Friedmann Robertson Walker universe composed of several perfect fluids. We consider the power law inflation and determine the equation of state parameters in terms of the parameters of modified gravity's Lagrangian in the early universe. We also discuss briefly the gravitational baryogenesis in this model.
\end{abstract}

\section{Introduction}

Modified theory of gravity, constructed by adding geometrical correction terms to the usual Einstein Hilbert Lagrangian has been used to study the inflationary epoch and the subsequent reheating stage in the early universe [1, 2. In this view the effective Lagrangian, $L$, in the early universe includes higher order curvature terms and inflation may be a natural result of this theory. In [1, it was shown that involving a term proportional to the square of the scalar curvature, i.e., $L=R+\alpha R^{2}$, results in a quasi-de Sitter expansion. In this model the Hubble parameter decreases slowly for large $\alpha$ before going into an oscillation phase which can reheat the universe [2]. In principle, one can assume that the effective Lagrangian is a function of the scalar curvature $L=f(R)$ 3. The time dependence of the scale factor depends on $f(R)$, e.g., if one chooses $f(R)=R+\alpha R^{2}+\beta R^{3}$, instead of an exponential like inflation, he may obtain a power law expansion for the universe [4. Some of particle physics problems such as the hierarchy problem [5] and baryogenesis [6] can also be studied in the framework of modified theory of gravity. Recently, the modified theory of gravity which is able to describe the present cosmic acceleration [7] without involving exotic dark energy [8] has attracted more attention.

In this paper we aim to study the universe in an era when the expansion can be described by the scale factor $a(t) \propto t^{\lambda}$. This assumption is consistent with most parts of the (Friedmann Robertson Walker expanding) universe history, when one of the components such as (dark) matter or radiation dominates. But in the framework of the usual theory of gravity, and in the presence of ordinary

*mohseni@phymail.ut.ac.ir 
matter and radiation, the power law expansion can not describe the inflationary and accelerating expansion phases. Besides, the gravitational baryogenesis proposal fails to determine the baryon asymmetry in radiation dominated era. In this paper we consider the modified theory of gravity, characterized by $f(R)=\sum_{i} A_{i} R^{n_{i}}$, in the early universe. We assume that the universe is composed of different perfect fluid components with constant equation of state parameters. Physical parameters of the system such as radiation temperature and the equation of state parameters of the fluids are determined in terms of $n_{i}$ 's and $A_{i}$ 's. At the end we use our results to determine the gravitational baryogenesis in a universe composed of two components in the framework of modified theory of gravity. Through the paper we use the units $c=k_{B}=1$.

\section{Power law expansion in modified theory of gravity}

We consider the modified theory of gravity described by the action

$$
S=\int\left(\frac{1}{16 \pi G} f(R)+L_{m}\right) \sqrt{-g} d^{4} x,
$$

where $L_{m}$ is the Lagrangian corresponding to the matter such as radiation, baryonic matter, dark matter and so on. The geometry of the universe which is assumed to be spatially flat, homogeneous and isotropic is described by Friedmann Robertson Walker (FRW) metric

$$
d s^{2}=-d t^{2}+a^{2}(t)\left(d x^{2}+d y^{2}+d z^{2}\right)
$$

$a(t)$ is the scale factor in terms of which the Hubble parameter is $H=\frac{a(t)}{a(t)}$. The Ricci scalar is

$$
R=6\left(\dot{H}+2 H^{2}\right) .
$$

By variation of the action with respect to the metric, we obtain

$$
g_{\mu \nu} \square f^{\prime}(R)-\nabla_{\mu} \nabla_{\nu} f^{\prime}(R)+f^{\prime} R_{\mu \nu}-\frac{f(R)}{2} g_{\mu \nu}=8 \pi G T_{\mu \nu}^{m},
$$

where $T_{\mu \nu}^{m}$ are the energy momentum tensor components of matter fields which behave like a perfect fluid. The energy density, $\rho$, and the pressure, $P$, may be derived from (4):

$$
\begin{aligned}
8 \pi G \rho= & \frac{f(R)}{2}+3 \frac{\dot{a}}{a} f^{\prime \prime}(R) \dot{R}-3 \frac{\ddot{a}}{a} f^{\prime}(R) \\
8 \pi G P= & -\frac{f(R)}{2}+\left(2 \frac{\dot{a}^{2}}{a^{2}}+\frac{\ddot{a}}{a}\right) f^{\prime}(R)-2 \frac{\dot{a}}{a} f^{\prime \prime}(R) \dot{R}-f^{\prime \prime \prime}(R) \dot{R}^{2}- \\
& f^{\prime \prime}(R) \ddot{R} .
\end{aligned}
$$

The energy conservation relation,

$$
\dot{\rho}+3 H(P+\rho)=0,
$$

is not independent of (5), but is required for consistency. We assume that the perfect fluid is effectively composed of non-interacting components, although 
each component may include subcomponents whose interactions are rapid compared to the expansion rate keeping them in thermal equilibrium. In this way the total energy density and the total pressure are given by

$$
\rho=\sum_{i} \rho_{i}, \quad P=\sum_{i} P_{i} .
$$

Each component satisfies

$$
\dot{\rho}_{i}+3 H\left(P_{i}+\rho_{i}\right)=0 .
$$

For time independent equation of state parameters (EOS) (denoted by $\gamma_{i}{ }^{\prime}$ s), the solution of (8), in terms of the scale factor, is

$$
\rho_{i}(t)=\rho_{i}\left(t_{0}\right)\left(\frac{a(t)}{a\left(t_{0}\right)}\right)^{-3\left(\gamma_{i}+1\right)} .
$$

Note that even for constant $\gamma_{i}$ 's, the EOS parameter of the universe,

$$
\gamma=\frac{P}{\rho}=\frac{\sum_{i} \gamma_{i} \rho_{i}}{\sum_{i} \rho_{i}}
$$

is time dependent. To study (5), let us take

$$
f(R)=\sum_{i} f_{i}(R),
$$

such that $f_{i}$ satisfies

$$
\begin{aligned}
8 \pi G \rho_{i} & =\frac{f_{i}(R)}{2}+3 \frac{\dot{a}}{a} f_{i}^{\prime \prime}(R) \dot{R}-3 \frac{\ddot{a}}{a} f_{i}^{\prime}(R) \\
8 \pi G P_{i} & =-\frac{f_{i}(R)}{2}+\left(2 \frac{\dot{a}^{2}}{a^{2}}+\frac{\ddot{a}}{a}\right) f_{i}^{\prime}(R)-2 \frac{\dot{a}}{a} f_{i}^{\prime \prime}(R) \dot{R}-f_{i}^{\prime \prime \prime}(R) \dot{R}^{2} \\
-f_{i}^{\prime \prime}(R) \ddot{R} . &
\end{aligned}
$$

In this way if (12) is satisfied, then (5) will be also satisfied. In this paper we restrict ourselves to the models characterized by [9]

$$
f(R)=\sum_{i} A_{i} R^{n_{i}} .
$$

$A_{i}$ 's and $n_{i}$ 's are real constants ( $n_{i}$ 's are not restricted to integer numbers). Note that $f(R)$ broken power law models such as [10]

$$
f(R)=R-m^{2} \frac{c_{1}\left(\frac{R}{m^{2}}\right)^{n}}{c_{2}\left(\frac{R}{m^{2}}\right)^{n}+1},
$$

where $m, n>0, c_{1}, c_{2}$ are real numbers, can be casted to (13) at high curvature limit $R \gg m^{2}$.

$f(R)$ in (1) must satisfy some stability conditions [1]. For the model (1), the condition $\frac{d^{2} f(R)}{d R^{2}}>0$, which in our model reduces to

$$
\sum_{i} n_{i}\left(n_{i}-1\right) A_{i} R^{n_{i}-2}>0,
$$


is necessary for classical stability of FRW solution in high-curvature regime. In the context of quantum mechanics this condition ensures the absence of tachyonic scalarons [1]. We also require that $\frac{d f(R)}{d R}>0$ or

$$
\sum_{i} n_{i} A_{i} R^{n_{i}-1}>0
$$

to prevent the graviton from turning into a ghost [11.

In the following We use the ansatz $a(t) \propto t^{\lambda}$ for the scale factor. This ansatz is allowed when the number of (dominant) fluid components is the same as the number of the terms in $f(R)$. In general, $\lambda$ depends on EOS parameters of the components of the perfect fluid, $\gamma_{i}$ 's, and $n_{i}$ 's. Now let us find the conditions required for consistency of $a(t) \propto t^{\lambda}$ with (12). By substituting the Ricci scalar, $R=6 \lambda \frac{2 \lambda-1}{t^{2}}$, and (91) into (12) we obtain

$$
(2 \lambda-1)^{n_{i}-1}(6 \lambda)^{n_{i}}\left(n_{i}(3-\lambda)-2 n_{i}^{2}-1+2 \lambda\right) A_{i} t^{-2 n_{i}}=16 \pi G \rho_{i}\left(t_{0}\right)\left(\frac{t}{t_{0}}\right)^{-3 \lambda\left(\gamma_{i}+1\right)} .
$$

The above equation is true for all $t$, provided that

$$
\frac{3 \lambda}{2}=\frac{n_{i}}{1+\gamma_{i}}=\frac{n_{j}}{1+\gamma_{j}}, \forall i, j
$$

and

$$
A_{i}=\frac{\rho_{i}\left(t_{0}\right) t_{0}^{3 \lambda\left(\gamma_{i}+1\right)}}{\delta_{i}}
$$

where

$$
\delta_{i}=\frac{3}{8 \pi G}\left(n_{i}(-\lambda+3)-2 n_{i}^{2}+2 \lambda-1\right)(6(2 \lambda-1))^{n_{i}-1} \lambda^{n_{i}} .
$$

Hence if the EOS parameters of the fluid components satisfy (17), and $A_{i}$ 's are given by (18), then in $f(R)$ gravity specified by (13), $a(t) \propto t^{\lambda}$ can be considered as the scale factor. If $n_{l}=0$, then we must take $\gamma_{l}=-1$ describing a cosmological constant corresponding to the vacuum energy. For positive $\lambda$, $n_{i}<0$ leads to $\gamma_{i}<-1$ describing a phantom like dark energy component.

The above discussion can be generalized to the case that $\lambda$ is a slowly varying function of time, $\lambda=\lambda(t), \lambda(t) t \ll \lambda(t)$ [9]. Time independence of $A_{i}$ requires

$$
\frac{\dot{\rho}_{i}}{\rho_{i}}=\frac{\left(n_{i}-2\right) \dot{R}}{R}+\frac{\dot{Q_{i}}}{Q_{i}}
$$

where $Q_{i}$ is defined through $Q_{i}=R^{2}-6 n_{i}\left(\dot{H}+H^{2}\right) R+6 n_{i}\left(n_{i}-1\right) H \dot{R}$. By $\gamma_{i}=-1-\frac{\rho_{i}}{3 H \rho_{i}}$ and (20), we can determine the time dependent EOS parameters

$$
\begin{aligned}
\gamma_{i}= & -\frac{3 \lambda-2 n_{i}}{3 \lambda}+\frac{4(4 \lambda-1) n_{i}^{2}+\left(4 \lambda^{2}-17 \lambda+2\right) n_{i}+11 \lambda-8 \lambda^{2}}{3 \lambda(2 \lambda-1)\left(2 n_{i}^{2}+(\lambda-3) n_{i}+(1-2 \lambda)\right)} \frac{\dot{\lambda} t}{\lambda} \\
& +\mathcal{O}\left(\frac{\dot{\lambda}^{2} t^{2}}{\lambda^{2}}\right) .
\end{aligned}
$$


Up to $\mathcal{O}\left(\frac{\dot{\lambda} t}{\lambda}\right)$, the relation between $\gamma_{i}$ 's may be obtained in a compact form

$$
\begin{aligned}
\gamma_{i}(t) & =\frac{n_{i}}{n_{j}} \gamma_{j}(t)+\left(\frac{n_{i}}{n_{j}}-1\right), \forall i, j \\
& =-1+\frac{2 n_{i}}{3 \lambda(t)} .
\end{aligned}
$$

It is worth to note that in a one component fluid, with EOS parameter $\gamma$, we have $\gamma=\frac{2 n}{3 \lambda}-1$, hence if the universe is approximately filled with radiation (i.e $\left.\gamma=\frac{1}{3}\right)$ then $\lambda=\frac{n}{2}$ and $R=3 n\left(\frac{n-1}{t^{2}}\right)$. Therefore for $n \neq 1$ we have $R \neq 0$ and $\dot{R} \neq 0$ while in Einstein theory of gravity we obtain $\dot{R}=0$. This is the note used in [6] to show that in modified gravity the gravitational baryogenesis may occur even in radiation dominated epoch.

Now assume that one of the thermal fluid components (e.g. the radiation component) has temperature $T$, i.e., the subcomponents of this component have nearly common temperature $T$. We assume also that the density of each component is given by

$$
\rho_{i}=\epsilon_{i} T^{\theta_{i}} .
$$

This is allowed when $T$ is proportional to a power of the scale factor. It is worth to note that $T$ may not be the temperature of other noninteracting fluid components. (9) and (18) can be used to obtain the time dependence of $T$

$$
T=\left(\delta_{i} \frac{A_{i}}{\epsilon_{i}}\right)^{\frac{1}{\theta_{i}}} t^{\frac{-2 n_{i}}{\theta_{i}}} .
$$

This equation holds for each $i$, therefore

$$
\frac{n_{i}}{\theta_{i}}=\frac{n_{j}}{\theta_{j}} \forall i, j .
$$

Besides, for $\forall i, j$ we must have

$$
\left(\frac{\delta_{i}}{\epsilon_{i}} A_{i}\right)^{\frac{1}{\theta_{i}}}=\left(\frac{\delta_{j}}{\epsilon_{j}} A_{j}\right)^{\frac{1}{\theta_{j}}}
$$

To elucidate our results, as an example, we assume that the universe is approximately composed of a radiation component (denoted by the subscript $\mathcal{R}$ ) and a non thermal component with EOS parameter $\omega[12$. These non-interacting components satisfy the energy conservation relation

$$
\begin{array}{r}
\dot{\rho_{\mathcal{R}}+4 H} \rho_{\mathcal{R}}=0 \\
\dot{\rho}_{\omega}+3 H(1+\omega) \rho_{\omega}=0 .
\end{array}
$$

The time derivative of the ratio of these components, denoted by $r:=\frac{\rho_{\mathcal{R}}}{\rho_{\omega}}$, is

$$
\dot{r}=3 H r\left(\omega-\frac{1}{3}\right)
$$

if $\omega>\frac{1}{3}, \dot{r}>0$ and $\rho_{\omega}$ component decreases more rapidly than radiation component and the universe will become radiation dominated. Following our previous discussions let us take

$$
f(R)=A_{\mathcal{R}} R^{n_{\mathcal{R}}}+A_{\omega} R^{n_{\omega}} .
$$


Stability conditions require that the parameters of the model satisfy

$$
n_{\mathcal{R}}\left(n_{\mathcal{R}}-1\right) A_{\mathcal{R}} R^{n_{\mathcal{R}}}+n_{\omega}\left(n_{\omega}-1\right) A_{\omega} R^{n_{\omega}}>0,
$$

and

$$
n_{\mathcal{R}} A_{\mathcal{R}} R^{n_{\mathcal{R}}-1}+n_{\omega} A_{\omega} R^{n_{\omega}-1}>0 .
$$

If the scale factor is given by $a(t) \propto t^{\lambda}$, then

$$
n_{\mathcal{R}}=2 \lambda, \quad n_{\omega}=\frac{3}{2}(1+\omega) \lambda .
$$

As a result, in this model, $\omega$ can be expressed in terms of $n_{i}$ 's: $\omega=\frac{4 n_{\omega}}{3 n_{\mathcal{R}}}-1$. The stability conditions is satisfied by choosing appropriate parameters for the model, e.g., if we take $n_{\omega}=1$, which results in $\omega=\frac{2}{3 \lambda}-1$, (29) reduces to $f(R)=A_{\omega} R+A_{\mathcal{R}} R^{n_{\mathcal{R}}}$ (the stability of models including this specific case was discussed in [13]) and the stabilities conditions become

$$
\begin{aligned}
& n_{\mathcal{R}}\left(n_{\mathcal{R}}-1\right) A_{\mathcal{R}} R^{n_{\mathcal{R}}}>0 \\
& n_{\mathcal{R}} A_{\mathcal{R}} R^{n_{\mathcal{R}}-1}+A_{\omega}>0 .
\end{aligned}
$$

For positive curvature and for $\lambda$ 's belonging to the domain $0.5<\lambda<0.64, A_{\mathcal{R}}$ becomes a positive real number (see (19) and (36)). Besides, $\delta_{\omega}=\frac{3 \lambda^{2}}{8 \pi G}$ implies that $A_{\omega}$ is positive. Therefore the stability conditions are satisfied. Note that in [14] it was proposed that $f(R)$ models with $B<0$, where $B$ is defined through

$$
B \equiv \frac{\frac{d^{2} f}{d R^{2}}}{\frac{d f}{d R}} \frac{d R}{d \ln a}\left(\frac{d \ln H}{d \ln a}\right)^{-1},
$$

are unstable to linear perturbations at high curvature, this leads us to take $\lambda>0.5$ which is in in agreement with $0.5<\lambda<0.64$ proposed above.

Following (9) and (18), the energy densities can be obtained as

$$
\rho_{i}=\delta_{i} A_{i} t^{-3 \lambda\left(1+\gamma_{i}\right)} .
$$

For radiation component this yields

$$
\rho_{\mathcal{R}}=\frac{3}{8 \pi G}\left(-10 \lambda^{2}+8 \lambda-1\right) \lambda^{2 \lambda}(6(2 \lambda-1))^{2 \lambda-1} A_{\mathcal{R}} t^{-4 \lambda} .
$$

On the other hand the temperature of the radiation component is given [15]

$$
\rho_{\mathcal{R}}=\epsilon_{\mathcal{R}} T^{4},
$$

where $\epsilon_{\mathcal{R}}=\frac{\pi^{2}}{30} g_{\star}$ and $g_{\star}$ is the total degrees of freedom of effective massless particles contributing in radiation component. Hence, in (23), $\theta_{\mathcal{R}}=4$. By using (37) and (35) we find out

$$
T=\left(\frac{\delta_{\mathcal{R}} A_{\mathcal{R}}}{\epsilon_{\mathcal{R}}}\right)^{\frac{1}{4}} t^{-\frac{n_{\mathcal{R}}}{2}} .
$$

By substituting (38) into (35) one gets

$$
\rho_{\omega}=\delta_{\omega} A_{\omega}\left(\frac{\epsilon_{\mathcal{R}}}{\delta_{\mathcal{R}} A_{\mathcal{R}}}\right)^{\frac{n_{\omega}}{n_{\mathcal{R}}}} T^{4 \frac{n_{\omega}}{n_{\mathcal{R}}}}
$$


By considering the assumption (23) we find

$$
\epsilon_{\omega}=\delta_{\omega} A_{\omega}\left(\frac{\epsilon_{\mathcal{R}}}{\delta_{\mathcal{R}} A_{\mathcal{R}}}\right)^{\frac{n_{\omega}}{n_{\mathcal{R}}}}, \theta_{\omega}=4 \frac{n_{\omega}}{n_{\mathcal{R}}} .
$$

If at a time denoted by $t=t_{R D}$, the energy density of radiation component becomes equal to the other component:

$$
\rho_{\omega}\left(t_{R D}\right)=\rho_{\mathcal{R}}\left(t_{R D}\right)=\epsilon_{\mathcal{R}} T_{R D}^{4},
$$

where $T_{R D}$ is the radiation temperature at $t_{R D}$, then the solution of (27) may be written as

$$
\rho_{\omega}=\epsilon_{\mathcal{R}} T_{R D}^{4}\left(\frac{T}{T_{R D}}\right)^{4 \frac{n_{\omega}}{n_{\mathcal{R}}}} .
$$

The temperature $T_{R D}$ can be determined in terms of the parameters of the model:

$$
T_{R D}=\epsilon_{\mathcal{R}}^{-\frac{1}{4}}\left(\delta_{\omega} A_{\omega}\right)^{\frac{n_{\mathcal{R}}}{4\left(n_{\mathcal{R}}-n_{\omega}\right)}}\left(\delta_{\mathcal{R}} A_{\mathcal{R}}\right)^{\frac{n_{\omega}}{4\left(n_{\omega}-n_{\mathcal{R}}\right)}} .
$$

As an application, we can use these results to study a simple gravitational baryogenesis model 12 , in the context of modified theory of gravity in the early universe composed approximately of radiation and a nonthermal component described by (27). The key ingredient in this theory is the coupling of derivative of the Ricci scalar curvature and the baryon number current

$$
\frac{\varepsilon}{\Lambda^{2}} \int d^{4} x \sqrt{-g}\left(\partial_{\mu} R\right) J^{\mu}
$$

where $\Lambda$ is a cutoff characterizing the scale of the energy in the effective theory and $\varepsilon= \pm 1$. (44) dynamically violates CPT giving rise to the baryon asymmetry. In a universe with spatially constant $R$, to obtain the chemical potential for baryon $\left(\mu_{B}\right)$ and antibaryons $\left(\mu_{\bar{B}}\right)$, we use

$$
\frac{1}{\Lambda^{2}}\left(\partial_{\mu} R\right) J^{\mu}=\frac{1}{\Lambda^{2}} \dot{R}\left(n_{B}-n_{\bar{B}}\right),
$$

where $n_{B}$ and $n_{\bar{B}}$ are the baryon and antibaryon number densities respectively. Therefore there is an energy shift, $\frac{2 \varepsilon \dot{R}}{\Lambda^{2}}$, for a baryon with respect to an antibaryon. We can assign a chemical potential to baryons: $\mu_{B}=-\mu_{\bar{B}}=-\frac{\varepsilon \dot{R}}{\Lambda^{2}}$. So, in thermal equilibrium there will be a nonzero baryon number density given by:

$$
n_{b}=n_{B}-n_{\bar{B}}=\frac{g_{b} T^{3}}{6 \pi^{2}}\left(\pi^{2} \frac{\mu_{B}}{T}+\left(\frac{\mu_{B}}{T}\right)^{3}\right),
$$

where $g_{b} \sim \mathcal{O}(1)$ is the number of internal degrees of freedom of baryons. The entropy density of the universe is given by $s=\frac{2 \pi^{2}}{45} g_{s} T^{3}$, where $g_{s} \simeq 106$ indicates the total degrees of freedom for relativistic particles contributing to the entropy of the universe [15]. In the expanding universe the baryon number violation decouples at a temperature denoted by $T_{D}$ and a net baryon asymmetry remains. The ratio $\frac{n_{b}}{s}$ in the limit $T_{D} \gg m_{b}$ ( $m_{b}$ indicates the baryon mass), and $T_{D} \gg \mu_{b}$ is then:

$$
\frac{n_{b}}{s} \simeq-\left.\varepsilon \frac{15 g_{b}}{4 \pi^{2} g_{s}} \frac{\dot{R}}{\Lambda^{2} T}\right|_{T_{D}} .
$$


Introduction of $\varepsilon$ gives us the possibility to choose the appropriate sign for $n_{b}$. In our model $\dot{R}$ is determined as

$$
\dot{R}=-6 n_{\mathcal{R}}\left(1-n_{\mathcal{R}}\right)\left(\frac{\epsilon_{\mathcal{R}}}{\delta_{\mathcal{R}} A_{\mathcal{R}}}\right)^{\frac{3}{2 n_{\mathcal{R}}}} T_{D}^{\frac{6}{n_{\mathcal{R}}}} .
$$

To derive (48) we have used the fact that the decoupling time is

$$
t_{D}=\left(\frac{\epsilon_{\mathcal{R}}}{\delta_{\mathcal{R}} A_{\mathcal{R}}}\right)^{-\frac{1}{2 n_{\mathcal{R}}}} T_{D}^{-\frac{2}{n_{\mathcal{R}}}}
$$

By putting (48) into (47) we can determine the baryon asymmetry

$$
\frac{n_{B}}{s} \sim \varepsilon \frac{0.02}{\Lambda^{2}} n_{\mathcal{R}}\left(1-n_{\mathcal{R}}\right)\left(\frac{\epsilon_{\mathcal{R}}}{\delta_{\mathcal{R}} A_{\mathcal{R}}}\right)^{\frac{3}{2 n_{\mathcal{R}}}} T_{D}^{\frac{6-n_{\mathcal{R}}}{n_{\mathcal{R}}}} .
$$

Note that (50) is valid for radiation dominated epoch as well as for non-radiation dominated era provided that the EOS parameter of non radiation component satisfies (32).

\section{References}

[1] A. A. Starobinsky, Phys. Lett. B 91, 99 (1980).

[2] M.B. Mijic, M. S. Morris and W. Suen, Phys. Rev. D 34, 2934 (1986); P. A. Anderson and W. Suen, Phys. Rev. D 35, 2940 (1987).

[3] J. D. Barrow and A. C. Ottewill, J. Phys. A 162757 (1983); J. D. Barrow and S. Cotsakis, Phys. Lett. B 214, 515 (1988).

[4] A. L. Berkin and K. Maeda, Phys. Lett. B 245, 348 (1990); J. D. Barrow and S. Cotsakis, Phys. Lett. B 232172 (1989); A. R. Liddle and F. Mellor, Gen. Rel. and Grav. 24, 897 (1992); E. Bruning, D. Coule and C. Xu, Gen. Rel. Grav. 26, 1197 (1994).

[5] S. Nojiri and S. D. Odintsov, Int. J. Geom. Meth. Mod. Phys. 4, 115 (2007).

[6] G. Lambiase and G. Scarpetta, Phys. Rev. D 74, 087504 (2006).

[7] A. G. Riess et al., Astron. J. 116, 1009 (1998); S. Perlmutter et al., Nature (London) 391, 51 (1998); P. M. Garnavich et al., Ap. J. 509, 74 (1998); S. Perlmutter et al., Astrophys. J. 517, 565 (1999).

[8] S. Nojiri and S. D. Odintsov, Phys. Lett. B 576, 5 (2003); D. N. Vollick, Phys. Rev. D 68 , 063510 (2003); K. Kleidis, A Kuiroukidis and D. B. Papadopoulos, Phys. Lett. B 546, 112 (2002); X. Meng and P. Wang, Class. Quant. Grav. 20, 4949 (2003); S. Nojiri and S.D. Odintsov, Phys. Rev. D 68, 123512 (2003); D. A. Easson, Int. J. Mod. Phys. A 19, 5343 (2004); A. D. Dolgov and M. Kawasaki, Phys. Lett. B 573, 1 (2003); G. Allemandi, A. Borowiec and M. Francaviglia, Phys. Rev. D 70, 103503 (2004); S. M. Carroll, A. De Felice, V. Duvvuri, D. A. Easson, Mark 
Trodden and M. S. Turner, Phys. Rev. D 71, 063513 (2005); T. Clifton and J. D. Barrow, Class. Quant. Grav. 23, 2951 (2006); T. Multamaki and I. Vilja, Phys. Rev. D 73, 024018 (2006); J. A. R. Cembranos, Phys. Rev. D 73, 064029 (2006); I. Brevik, Int. J. Mod. Phys. D 15, 767 (2006); K. Koyama, J. Cosmol. Astropart. Phys. 0603 (2006) 017; L. Amendola, D. Polarski and S. Tsujikawa Phys. Rev. Lett. 98, 131302 (2007); S. Tsujikawa, Phys. Rev. D 76, 023514 (2007); L. Amendola and S. Tsujikawa, arXiv: 0705.0396 [astro-ph]; K. Atazadeh and H. R. Sepangi, Int. J. Mod. Phys. D 16, 687 (2007); S. Rahvar and Y. Sobouti, arXiv: 0704.0680; H. Mohseni Sadjadi, Phys. Rev. D 76, 104024 (2007); F. Briscese, E. Elizalde, S. Nojiri and S. D. Odintsov, Phys. Lett. B 646, 105 (2007); B. Jain and P. Zhang, arXiv:0709.2375v1 [astro-ph]; S. Wang, L. Hui, M. May and Z. Haiman, Phys. Rev. D 76, 063503 (2007); M. Fairbairn and S. Rydbeck, arXiv: astro-ph/0701900v2; A. De Felice, P. Mukherjee and Y. Wang, arXiv: 0706.1197v1 [astro-ph]; J. W. Moffat and V. T. Toth, arXiv: 0710.0364v2 [astro-ph]; C. Charmousis, R. Gregory and A. Padilla, arXiv: 0706.0857v1 [hep-th].

[9] S. Nojiri and S. D. Odintsov, arXiv:hep-th/0611071v2.

[10] W. Hu and I. Sawicki, arXiv:0705.1158v1 [astro-ph]; S. Nojiri and S. D. Odintsov, arXiv:0710.1738v2 [hep-th].

[11] I. Sawicki and W. Hu, Phys. Rev. D 75127502 (2007); A. A. Starobinsky, JETP Lett. 86, 157 (2007), Pisma Zh. Eksp. Teor. Fiz. 86, 183 (2007); L. Pogosian and A. Silvestri, arXiv:0709.0296v2 [astro-ph].

[12] H. Davoudiasl, R. Kitano, G. D. Kribs, H. Murayama and P. J. Steinhardt, Phys. Rev. Lett. 93, 201301 (2004); H. Mohseni Sadjadi, arXiv:0709.0697v1 [gr-qc].

[13] S. Capozziello, S. Nojiri, S.D. Odintsov and A. Troisi, Phys. Lett. B 639 135 (2006).

[14] Yong-Seon Song, W. Hu and I. Sawicki, Phys. Rev. D 75044004 (2007).

[15] E. W. Kolb and M. S. Turner, The Early Universe, Westview Press, (1994). 